\title{
Minimal Spanning Tree Graphs and Power Like Scaling in FOREX Networks
}

\author{
A.Z. Górski ${ }^{a}$, S. Drożd $\dot{Z}^{a, b}$, J. KWAPIEŃ ${ }^{a}$ \\ AND P. OŚWIȨCIMKA ${ }^{a}$ \\ ${ }^{a}$ H. Niewodniczański Institute of Nuclear Physics, Polish Academy of Sciences \\ Radzikowskiego 152, 31-342 Kraków, Poland \\ ${ }^{b}$ Institute of Physics, University of Rzeszów \\ 35-310 Rzeszów, Poland \\ Correlation matrices of foreign exchange rate time series are investi- \\ gated for 60 world currencies. Minimal spanning tree graphs for the gold, \\ silver and platinum are presented. Inverse power like scaling is discussed for \\ these graphs as well as for four distinct currency groups (major, liquid, less \\ liquid and non-tradable). The worst scaling was found for USD and related \\ currencies.
}

PACS numbers: 89.65.Gh, 89.75.Fb, 05.45.Tp

\section{Introduction}

The foreign currency exchange (FOREX, FX) market is the world largest financial market and the exchange rates have direct influence on all other markets because the price of any asset is expressed in terms of a currency. The FX market is strongly decentralized (transactions take place in many different places in the world), commonly accessible and extremely difficult to control. In addition, there is no friction (transactions are basically commission free). Due to time differences FX transactions are performed 24 hours a day, 5.5 day a week with maximum between 1 and 4 p.m. GMT, when both American and European markets are open. Hence, the FX time series are especially worth of detailed analysis. The FX market can be viewed as a complex network of mutuallyinteracting nodes, each node being an exchange rate of two currencies and being strongly interconnected with complex nonlinear interactions to other nodes. Any currency can be expressed in terms of a given currency that is called the base currency.

\section{Correlation matrix}

For a financial time series of an $i$-th asset $(i=1, \ldots, n)$ at time $t, x_{i}\left(t_{i}\right)=x_{i}$, one defines its return over the time period $\tau$ as $G_{i}(t ; \tau)=\ln x_{i}(t+\tau)-\ln x_{i}(t)$, 
where the logarithm is used to have additivity with respect to the return time $\tau$. For the FX series instead of a value $x_{i}(t)$ one has $x_{A}^{B}(t)$, an exchange rate, i.e. a value of currency $A$ expressed in terms of a base currency $B$ and instead of $x_{i}(t)$ one has $x_{A}^{B}(t)$. The returns can be denoted as $G_{A}^{B}(t)$ and they are clearly antisymmetric: $G_{A}^{B}(t ; \tau)=-G_{B}^{A}(t ; \tau)$ and they fulfill the triangle rule: $G_{A}^{B}(t ; \tau)+$ $G_{B}^{C}(t ; \tau)+G_{C}^{A}(t ; \tau)=0[1]$, already for relatively small values of $\tau$. As a result, for a set of $n$ currencies we have $N=n-1$ independent values and the same number of nodes with a given base currency.

We study time series of daily data for 60 currencies, including gold, silver and platinum [2]. The data were taken for the time period Dec 1998-May 2005 and the series were filtered to get rid of misprints. In particular, we have removed daily jumps greater than $5 \sigma$ (less than $0.3 \%$ of data points). Also, the gaps related to non-trading days were synchronized. For each exchange rate we have obtained a time series of 1657 data points. The currencies are denoted according to ISO 4217 standard, and they can be divided into four groups, according to their liquidity. The major currencies, we call the $A^{*}$ group, include USD, EUR, JPY, GBP, CHF, CAD, AUD, NZD, SEK, NOK, DKK (11 currencies). To the group $A$ belong all other liquid currencies: CYP, CZK, HKD, HUF, IDR, ILS, ISK, KRW, MXN, MYR, PHP, PLN, SGD, SKK, THB, TRY, TWD, XAG, XAU, XPT, ZAR (21 currencies). Less liquid currencies (group $B$ ) include: ARS, BGN, BRL, CLP, KWD, RON, RUB, SAR, TTD (9 currencies). Finally, the non-tradable currencies (group $C$ ) taken into account are: AED, COP, DZD, EGP, FJD, GHC, HNL, INR, JMD, JOD, LBP, LKR, MAD, PEN, PKR, SDD, TND, VEB, ZMK (19 currencies). Exchange rates of these currencies can be viewed as a complex network of mutually interacting nodes.

The (symmetric) correlation matrix $(\mathrm{CM})$ can also be computed in terms of the normalized returns, $g_{A}(t)$. To this end one has to form $N$ time series $\left\{g_{A}^{X}\left(t_{0}\right), g_{A}^{X}\left(t_{0}+\tau\right), \ldots, g_{A}^{X}\left(t_{0}+(T-1) \tau\right)\right\}$ of length $T$. These series can form an $N \times T$ rectangular matrix $M$. The $\mathrm{CM}$ can then be rewritten in the matrix notation as

$$
C^{X} \equiv[C]_{A B}^{X}=\frac{1}{T} M^{X} \widetilde{M}^{X}
$$

where tilde stands for the matrix transposition. By construction the trace of the CM equals the number of time series $\operatorname{Tr} \boldsymbol{C}^{X}=N$.

Maximal eigenvalues for all base currencies considered are plotted in Fig. 1 [3]. The plot can be divided into three parts. The largest maximal eigenvalues correspond to the currencies that either have a very strong drift, independent of the behavior of other currencies (like GHC) or whose fluctuations are to large extent independent of the global $\mathrm{FX}$ behavior. In the medium range (approximately $\lambda_{N}=0.4 N \div 0.65 N$ ) one finds typical liquid currencies. Finally, the smallest values of $\lambda_{N}$ correspond to the USD and other currencies that are strongly tied to US dollar. It should be stressed that similar behavior of eigenvalues has been 
found for smaller subsectors of the analyzed currencies. In particular, we have also calculated spectra of tradable and non-tradable (type $C$ ) subsectors. Finally, the second largest eigenvalues $\left(\lambda_{N-1}\right)$ were calculated and they are not so clearly separated from the whole spectrum in two cases: (i) for currencies with very large $\lambda_{N}$ i.e. with strong independent drift, like GHC and (ii) for USD and currencies closely tied to it.

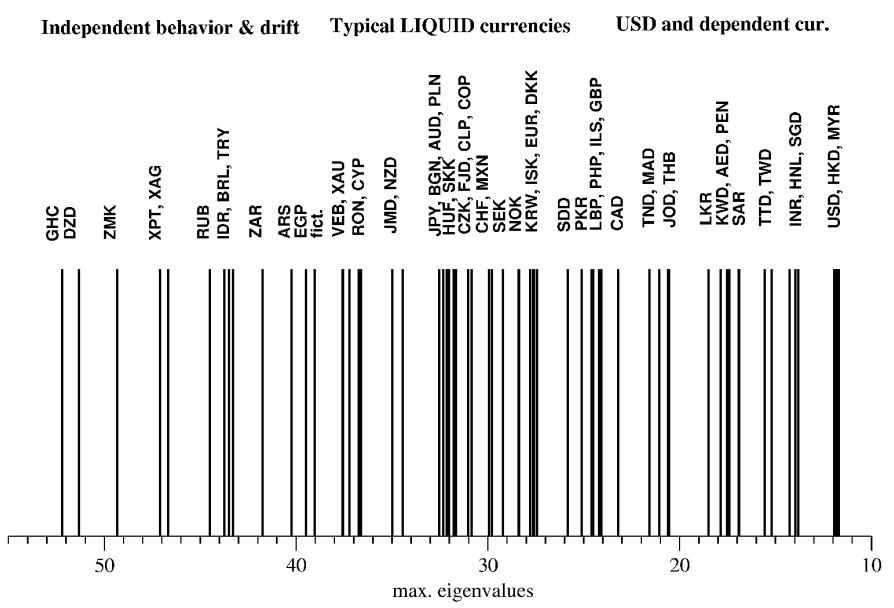

Fig. 1. Maximal eigenvalues of $\mathrm{CM}$ for all base currencies, including the fictitious (Gaussian noise) currency. Three types of behavior are indicated: independent drift, typical behavior and USD-tied currencies.

In addition to real currencies we have calculated two artificial cases. The first one, denoted as r.m. is for all the shuffled time series, with all time correlations destroyed. As a result we obtained CM spectrum almost identical as from the random matrix theory. In the second case we have assumed the Gaussian noise as the exchange rate of a fictitious currency (fict) to the USD. The exchange rates to all other currencies were computed via USD. Clearly, in this way the original time correlations of USD were modified. But due to homogeneity of Gaussian noise non-trivial time correlations did survive. In effect, the CM spectrum for our fictitious currency is quite similar to typical real currencies. In terms of Fig. 1 the maximal eigenvalue for this currency is close to the borderline between liquid and drifting currencies.

\section{Power scaling of minimal spanning tree graphs}

The minimal spanning tree (MST) graphs were introduced in graph theory quite long ago [4]. Later they were rediscovered several times $[5,6]$. To analyze the stock market correlations they were applied by Mantegna [7] and just recently for FX correlations $[1,8]$. However, in [8], due to small number of currencies, all graphs were connected in one bigger graph. 
To construct the MST graph we choose the following metric:

$$
d^{X}(A, B)=\sqrt{\left(1-C_{A B}^{X}\right) / 2}, \quad 0 \leq d^{X}(A, B) \leq 1,
$$

where $X$ denotes the base currency. The distance between two time series is smaller if their correlation coefficient is closer to unity. To each time series corresponds one node (vertex) in the graph. We connect two nodes, $A$ and $B$, with a line ("leg") if their distance $d^{X}(A, B)$ is the smallest. In the next step we look for another two closest nodes and again we connect them with a line. This procedure is repeated until we obtain a connected tree graph. The number of legs attached to a given node we call multiplicity of that node, that will be denoted by $K$. Clearly, multiplicity of a node is an integer number, $K<N$, where $N$ is total number of nodes in the MST graph. Sample MST graphs for FX time series were presented in $[1]$.

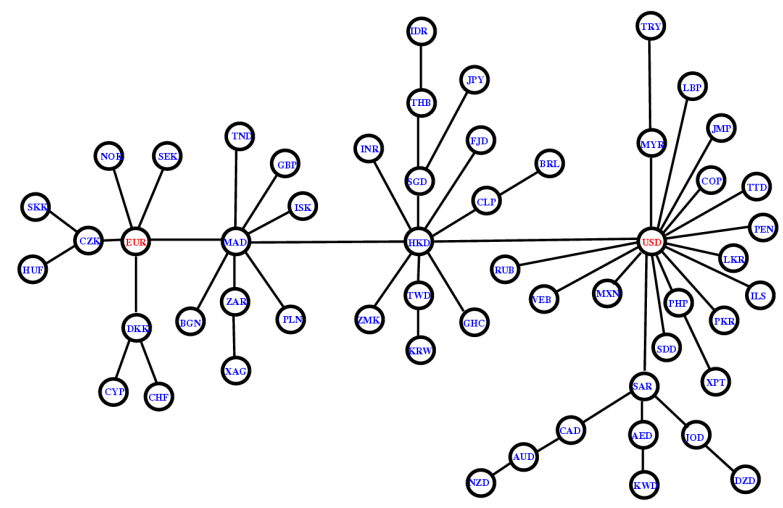

Fig. 2. MST graph for XAU taken as the base currency.

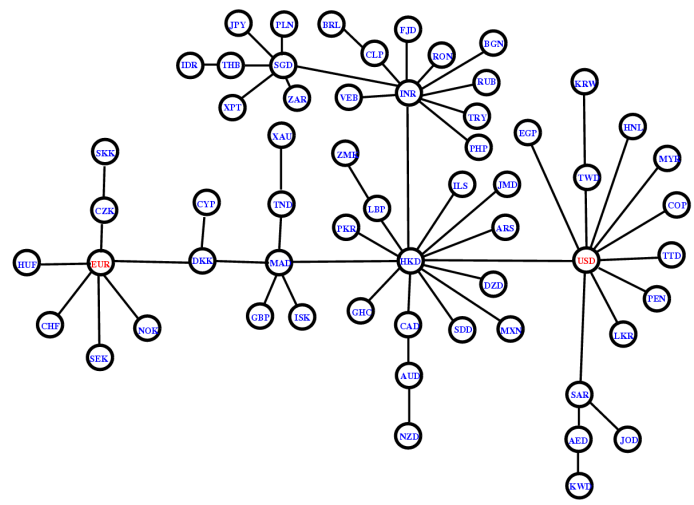

Fig. 3. MST graph for XAG taken as the base currency. 


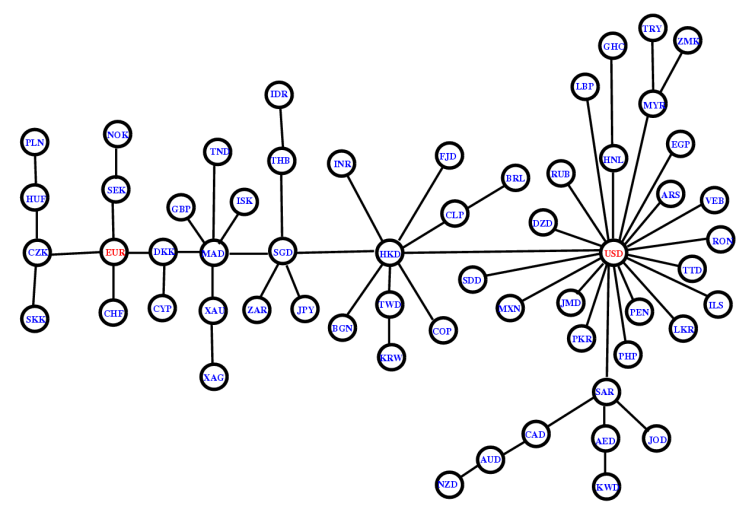

Fig. 4. MST graph for XPT taken as the base currency.

Figures 2-4 display MST graphs for gold, silver and platinum, the oldest traditional currencies. In all MST graphs for precious metals there is a high multiplicity USD node $(K \geq 10)$. This cluster is extended by the neighboring HKD cluster of considerable size $(K \geq 7)$. On the other end of the graph there is more modest EUR node (multiplicity $K=4 \div 6$ ) with European currencies. It is interesting to notice that while the currencies CZK, SKK and HUF have stable position in the euro node, the Polish currency has not. In case of gold (XAU) taken as the base currency, PLN is connected to Moroccan dirham (MAD) and for silver (XAG) it is connected to Singapore dollar (SGD). This indicates a more diversified role of PLN in the world financial system.

In our case we have $N=59$ nodes for a given base currency. Their multiplicity is denoted by $K_{A}$. By integer function $N^{\prime}(K)$ we denote number of nodes with exactly $K$ legs. Because the total number of nodes, $N$, is relatively small, we introduce the integrated quantity

$$
N(K)=\sum_{i=K}^{K_{\max }} N^{\prime}(K),
$$

where $K_{\max }$ denotes the maximal number of legs in the MST graph. $N(K)$ is the number of nodes with $K$ or more legs (a cumulative distribution with respect to $N^{\prime}(K)$ ). Clearly, $N(1)=N$.

To be able to compare graphs with different number of nodes it is convenient to introduce a normalized (and discrete) function $F(K)=N(K) / N$. For any MST graph we have $F(1)=1$. Counting legs in all nodes of a graph we can construct discrete functions $N(K)$ and $F(K)$.

Now, we are ready to investigate the functional form of the integer valued discrete function $N(K)$. We have found that on average it reveals a scale free, inverse power behavior. However, to estimate quality of the fit we must take into account that the function has integer values and the inverse power fit by construction cannot be perfect. The smallest possible deviation from a nearest 
integer value, $\delta(K)$, is always in the range $0 \leq \delta(K) \leq 1 / 2$. Hence, for the best fit we have the average deviation $\delta=\langle\delta(K)\rangle_{K}=1 / 4$. And for the corresponding normalized function

$$
\Delta F=\langle F(K)\rangle_{K}=\frac{\delta}{N} \approx 0.004
$$

Because by definition, $F(K) \leq 1 \mathrm{Eq}$. (4) implies that the relative error, in the statistical (large $N$ ) limit should be: $\Delta F / F>0.4 \%$. This result means that even in the case of very large number of legs one cannot obtain better fit than about half percent. In our case we have only 59 legs, a number far too small to have more than a few nodes with higher multiplicity. By inspection of graphs in Figs. 2-4 one can see only single nodes with multiplicities $\geq 10$. Hence, we are very far from what could be called the "large N limit" for the MST graphs. As a result, even for the best fits one should expect that its accuracy must be much worse than in the ideal case of half percent. Especially for the inverse power like fits, where the tail is relatively long and significant. In this case one can reasonably expect discrepancies around $5 \%$ for a good fit.

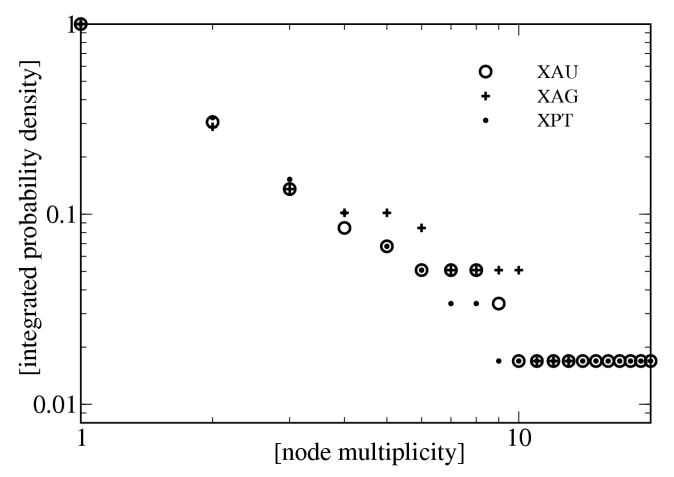

Fig. 5. Nodes multiplicity cumulative distribution for gold (XAU), silver (XAG) and platinum (XPT) taken as the base currency.

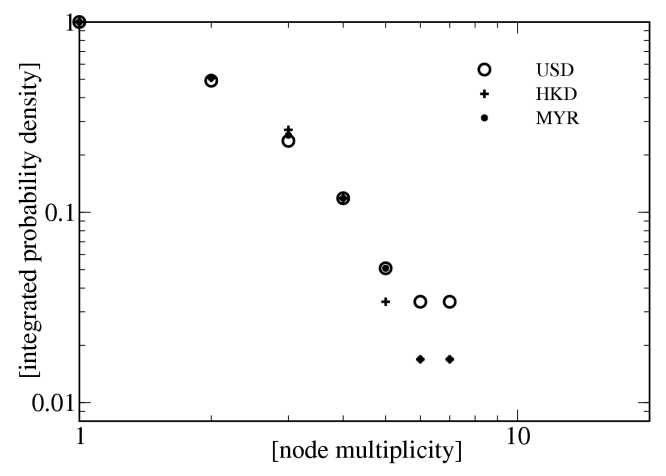

Fig. 6. Nodes multiplicity cumulative distribution for currencies with the worst power fits (USD, HKD and MYR). 


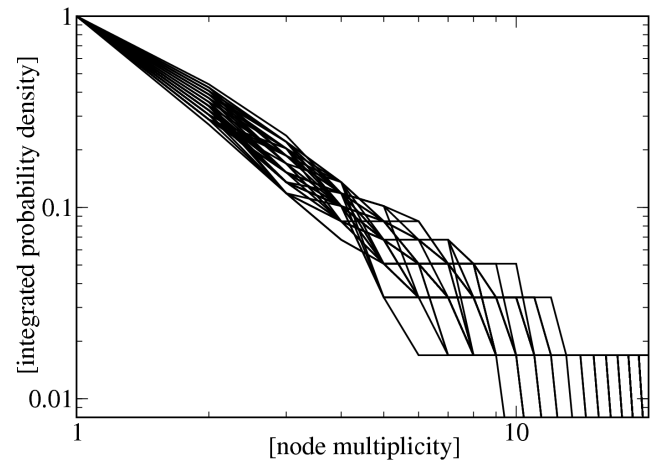

Fig. 7. Nodes multiplicity cumulative distribution for remaining 54 currencies taken as the base currency.

TABLE

Power fits for currencies of different groups: $A^{*}, A, B$, and $C$.

\begin{tabular}{c|c|c|c|c}
\hline \hline Base currency & $\alpha$ & $\Delta \alpha$ & $\Delta \alpha / \alpha$ & $\lambda_{N}$ \\
\hline metals & 1.42 & 0.09 & $6.5 \%$ & 43.7 \\
\hline$A^{*}$ & 1.43 & 0.08 & $5.4 \%$ & 32.1 \\
$A$ & 1.39 & 0.11 & $7.7 \%$ & 23.2 \\
$B$ & 1.34 & 0.08 & $6.0 \%$ & 29.9 \\
$C$ & 1.33 & 0.08 & $5.9 \%$ & 27.4 \\
\hline average & 1.43 & 0.09 & $6.3 \%$ & 27.3 \\
\hline r.m. & 2.33 & 0.63 & $27 \%$ & 1.4 \\
fict. & 1.55 & 0.08 & $5.4 \%$ & 39.0
\end{tabular}

Inverse power like fits for metals (XAU, XAG and XPT) and for the worst fits (USD, HKD and MYR) are shown in Figs. 5 and 6. In the latter case the tail (nodes of multiplicity $\geq 10$ ) is absent and this is the reason for bad fit. The currencies HKD and MYR are strongly connected to USD. In Fig. 7 the data for all 60 base currencies are plotted. With a few exceptions there is good power fit for nodes' multiplicity distribution. Detailed numerical data, including the average power exponent $\alpha$, its standard error $(\Delta \alpha)$, and average values of the maximal $\mathrm{CM}$ eigenvalues for all four groups of currencies $\left(A^{\star}, A, B\right.$, and $\left.C\right)$ are given in Table. The average value for $\alpha$ is equal to 1.43, and the relative errors are only slightly higher than suggested $5 \%$. For the worst fits (USD, HKD and MYR) the corresponding relative errors were found about $10 \%$ (USD) and higher (for HKD and MYR). In addition, results for the shuffled time series and thus destroyed correlations (case r.m.) and with the base currency being the Gaussian noise are 
given at the bottom of Table. In the former case, the error was about twice bigger as the worst fit for the real currencies - there is no scaling.

\section{Conclusion}

To summarize, it has been found that power like fit and scale free behavior is fairly good for majority of base currencies. The worst scaling, $\Delta \alpha / \alpha \geq 9 \%$, was obtained for USD and currencies tied to it (HKD, MYR, JMD, LBP, MAD). However, even in the worst case $(14 \%)$ this is much lower value than for the case of shuffled time series (r.m.), where the relative error is about $27 \%$. The reason is that taking USD as the base currency one eliminates its node from the MST graph. Hence, one of largest clusters (i.e. large multiplicity node) is missing and the tail of the distribution is getting thinner. Because of the relatively modest total number of nodes this effect is quite significant. The effect is more drastic for shuffled time correlations, where there is no power like tail at all. On the other hand, strong clustering of a currency means that it is influential for the FX market. Taking into account that the total number of nodes (number of currencies) cannot be very big, the standard error of order of a few percent should be considered as fairly low.

Similar power like scaling has been obtained by other authors for other complex networks, with the scaling exponents $\alpha$ in the range between 1.1 and 1.7 $[9,10]$. In our calculations the corresponding range is $1.3<\alpha<2.3$. However, for the two base currencies with $\alpha \geq 2$ (HKD and MYR) the power fit is not so good.

\section{References}

[1] A.Z. Górski, S. Drożdż, J. Kwapień, P. Oświęcimka, Acta Phys. Pol. B 37, 2987 (2006).

[2] Sauder School of Business, Pacific Exchange Rate System, http://fx.sauder.ubc.ca/data.html (2006).

[3] S. Drożdż, A.Z. Górski, J. Kwapień, Eur. Phys. J. B 58, 499 (2007).

[4] J. Kruskal, Proc. Am. Math. Soc. 7, 48 (1956).

[5] C.H. Papadimitrou, K. Steigliz, Combinatorial Optimization, Prentice-Hall, Englewood Cliffs 1982.

[6] D.B. West, Introduction to Graph Theory, Prentice-Hall, Englewood Cliffs 1996.

[7] R.N. Mantegna, Eur. Phys. J. B 11, 193 (1999).

[8] M. McDonald, O. Suleman, S. Williams, S. Howison, N.F. Johnson, Phys. Rev. E 72, 046106 (2005).

[9] R. Albert, A.-L. Barab'asi, Rev. Mod. Phys. 74, 47 (2002).

[10] S. Boccaletti, V. Latora, Y. Moreno, M. Chavez, D.U. Chwang, Phys. Rep. 424, 175 (2006). 